\title{
Learning to Interpret Spatial Natural Language in terms of Qualitative Spatial Relations
}

\author{
Parisa Kordjamshidi ${ }^{1}$, Joana Hois ${ }^{2}$, \\ Martijn van Otterlo ${ }^{1}$, and Marie-Francine Moens ${ }^{1}$ \\ ${ }^{1}$ Katholieke Universiteit Leuven, \\ Departement Computerwetenschappen, \\ parisa.kordjamshidi, martijn.vanotterlo, \\ sien.moens@cs.kuleuven.be \\ ${ }^{2}$ University of Bremen, Faculty of Computer Science, \\ joana@informatik.uni-bremen. de
}




\section{Summary}

Computational approaches in spatial language understanding nowadays distinguish and use different aspects of spatial and contextual information. These aspects comprise linguistic grammatical features, qualitative formal representations, and situational context-aware data. In this chapter, we apply formal models and machine learning techniques to map spatial semantics in natural language to qualitative spatial representations. In particular, we investigate whether and how well linguistic features can be classified and automatically extracted and mapped to region-based qualitative relations using corpus-based learning. We separate the challenge of spatial language understanding into two tasks: (i) we identify and automatically extract those parts from linguistic utterances that provide specifically spatial information, and (ii) we map the extracted parts that result from the first task to qualitative spatial representations. In this chapter, we present both tasks and we particularly discuss experimental results of the second part of mapping linguistic features to qualitative spatial relations. Our results show that region-based spatial relations can indeed be learned to a high degree and that they are distinguishable on the basis of different linguistic features. 


\section{Introduction}

The interpretation of natural language is essential in areas such as artificial intelligence (Roy and Reiter, 2005), computational linguistics (Kruijff et al., 2007), human-computer interaction (Nguyen and Wachsmuth, this volume), the semantic web (Fonseca and Rodriguez, 2007), and geographic information systems (Mani et al., 2008). More recently, the interplay between language and vision is investigated in-depth, as, for instance, the contributions by Avraamides et al., Barclay and Galton, Bhatt et al., Chown, and Nguyen and Wachsmuth (this volume) show. In all these fields, different formalisms have been developed that tackle the problem of interpreting natural language expressions in terms of their situation-based or contextualized meaning.

In this chapter, we address a specific aspect of spatial language interpretation as we focus on interpreting (English) spatial language by mapping its situational meaning to qualitative spatial representations (QSR). Our approach is motivated by the potential cognitive adequacy of its components as well as by technical benefits of the component distinctions. The diversity and variety of spatial language usage (for more detailed examples see Denis and Fernandez; Taylor and Brunyé; Seifert and Tenbrink, this volume) requires a flexible mechanism that allows a situation-based interpretation (Bateman, 2010a) resulting in a combination of different spatial 
components. Here, we concentrate on one component for representing spatial language in an adequate way and another component for representing qualitative spatial information, and we map the first to the second (Kordjamshidi et al., 2011a).

In our approach, we investigate the use of machine learning techniques for mapping spatial language to specific spatial qualitative representations. An intuitive argument for applying learning techniques is that it is a difficult task to formulate accurate models and rules that capture the entire knowledge necessary to extract spatial information from language. Humans, however, are able to easily understand and react to spatial language, and consequently, a suitable solution is the generation of many example sentences together with their spatial meanings by humans (i.e., annotated data), and to use this data for learning a mapping from linguistic sentences to specific spatial semantics automatically. This learning procedure can exploit any set of linguistic features that can be extracted from the sentences (e.g., dependency structure or parse tree) or from external resources, and - based on appropriately selected features - generalize to unseen sentences. The novel combination of techniques as put forward in this chapter aims at unifying the advantages from two sides, namely spatial information available from qualitative spatial representations as well as corpus-based information available from linguistic data.

Although the mapping from spatial language to QSR covers only parts of the entire spatial 
language interpretation process and spatial language is more flexible in its descriptions and expressions than QSR, this mapping is one central task in spatial language interpretation as spatial language describes spatial information primarily in qualitative terms. QSR may also lead to more efficient and effective reasoning strategies, i.e., the mapping from spatial language to a qualitative formal model can allow spatial reasoning with this model, whereas spatial reasoning with spatial language itself is hardly feasible (Bateman et al., 2010). Furthermore, QSR is often used in diverse domains and applications for spatial information (e.g., Bhatt et al., Chown, Seifert and Tenbrink, this volume), and our approach aims at providing the link between spatial natural language and the qualitative representations used within those tools and systems.

In contrast to our mapping approach, some spatial models exist that define spatial relations on the basis of linguistic terms (e.g., (Kurata and Shi, 2008)). Such models reflect, however, typically not spatial language semantics but specific meanings that are determined by the model's axioms. The diverse use of language requires yet more complex and flexible logical approaches (Bateman, 2010a). These approaches have to take into account contributing aspects for spatial language interpretations, such as context or world knowledge, in order to achieve a mapping from spatial language to qualitative representations (Hois and Kutz, 2008b; Kordjamshidi et al., 2010). 
Our spatial language interpretation approach automatizes the mapping from spatial language to QSR by using machine learning techniques and a holistic approach for describing spatial language. For this purpose, we distinguish two main levels (Bateman, 2010b):

(I) a linguistic level, in which natural language is analyzed and parts of a sentence, i.e., linguistic features are syntactically and semantically categorized as having different spatial roles that convey certain spatial information, and

(II) a formal level, in which the linguistic features are mapped to specific qualitative spatial representations, i.e., formal spatial calculi.

For example, in the sentence "The book is on the table", the first step is the identification of spatial linguistic features, e.g., a spatial relation "on" that holds between the "book" and the "table", and the second step is the mapping to an adequate spatial calculus, e.g., a connection calculus that formalizes an 'on'-relation. Both levels use symbolic spatial information for representing the linguistic features and qualitative models, and both levels use machine learning for extracting the linguistic features and mapping them to qualitative models. This allows us to formally structure and present spatial information based on available linguistic characteristics and to deal with aspects of ambiguity and uncertainty caused by linguistic underspecification, granularity differences, context dependencies, metaphors, etc. This is particularly important as we 
apply our approach to arbitrary linguistic sources, which is in contrast to using only a limited range of spatial language or phrases of which it is known that they contain spatial information (Kollar et al., 2010; Kelleher and Costello, 2009; Li et al., 2007).

In essence, the novelty of the presented work lies in the use of machine learning as a methodology for interpreting spatial language in terms of qualitative representations. We analyze three main aspects of this process: first, the performance of automatically mapping linguistic data to linguistic spatial features and to spatial semantics as well as to modalities of a linguistically motivated spatial ontology (GUM-Space); second, the feasibility of learning the mapping of linguistic spatial semantics to a formal spatial calculus, using the Region-Connection Calculus (RCC) as an example for topological information; and third, the interaction between different linguistic features and their contributing meaning during the overall process.

In the next section, we present specific aspects of spatial language that are necessary for understanding spatial language in terms of qualitative spatial calculi. Subsequently, we theoretically introduce our spatial language interpretation approach by presenting the different components of the mapping process from spatial language to spatial calculi. Here, we primarily employ a region-based calculus for topological information, though other types of spatial relations are integrated into our approach as well. Thereafter, we present experimental results and 
machine learning outcomes for mapping spatial language to RCC. Here, we also analyze those linguistic features with highest impact, address corpora acquisition and issues, and discuss domain-specific experimental effects. Finally, we conclude with discussions and future work.

\section{Learning to Understand Spatial Language}

Natural language can be used to communicate spatial information, e.g., spatial relations between objects and their relative location or change in space. For instance, the sentence "Give me the gray book on the big table" contains information about a spatial configuration of two objects '(book, table)' and an anticipated movement of the book. Different grammatical and semantic linguistic features play important roles in forming the meaning of spatial configurations.

A core task in spatial language understanding is to identify words or phrases with labels that express their spatial role in a sentence. For example, in the sentence "The gray book is on the big table”, the preposition on indicates a spatial relation between the gray book and the big table. However, not every preposition has a spatial meaning or only one spatial meaning, as lexical entries can generally have multiple meanings and multiple (spatial) roles. For instance, the preposition "on" in the example above has a spatial meaning but the preposition "on" in "I can count on him" has not. The following example (from (Bateman et al., 2010)) exemplifies such 
subtle notions.

(1) He left the institute an hour ago.

(2) He left the institute a year ago.

The first sentence describes a spatial relocation, in which a person leaves a building and goes somewhere else, i.e., the person is no longer in the building. The second sentence describes a situation of fundamental change beyond an exclusively spatial meaning, in which the person has apparently quit his job. Another set of examples is:

(3) The computer is on the table and the mouse is to the left of it.

(4) The party could be considered at the far left of the political spectrum.

Sentence 3 expresses two explicit physical relations about objects on a table. Sentence 4 uses a similar relation "at the far left of" though with a more conceptual meaning. It is possible to put the party on the left side of it only when explicitly drawing this "political spectrum" on a piece of paper. Thus, similar lexical items can give rise to very different spatial meanings, whereas two different descriptions can have similar or even identical semantics:

(5) Looking over his right shoulder he saw his dog sitting quietly. 
(6) The dog sat quietly on the floor to his right.

Another important aspect is that spatial information in sentence 1 and 2 is mainly conveyed by verbs, and in 3-6 by prepositions. Furthermore, some types of information are not explicitly given by the words but can be inferred, e.g., that the dog is back-right of the person in sentence 5 . Other linguistic efforts may be needed for such (spatial) inferences, for example the resolution of anaphora.

Despite the many variations in spatial information of natural language expressions, a useful abstraction is that a sentence is able to express spatial relations among objects. For example, sentence 6 expresses a relation TOTHERIGHTOF( $\log$,he $)$, in which we can distinguish different spatial roles: TOTHERIGHTOF expresses a relation, and $d o g$ and he are arguments of the relation with specific roles. Note, however, that this does not indicate the perspective, from which the relation TOTHERIGHTOF( $\operatorname{dog}$,he $)$ holds; this perspective information provides yet another spatial role. In this chapter, we use such spatial roles of linguistic elements in order to learn and extract them automatically from any given sentence. In order to apply machine learning effectively, a clear task definition as well as annotated data are required. Semantic hand-labeling of natural language, however, is an ambiguous, complex, and expensive task (Barclay and Galton, this volume). And in addition, to map the extracted linguistic features to qualitative representations, 
visual data referring to the same linguistic data is required to acquire respective qualitative spatial relations.

Automated spatial role extraction from language has been studied in only few applications, see, e.g., (Li et al., 2007) for Chinese languages. It focuses on fable stories, however, it only supports limited automatic recognition of spatial elements. The system presented in (Kollar et al., 2010) operates in a human-robot interaction context and follows natural language route directions. It is more flexible in extracting spatial information, though it expects only spatial language as input. Work by (Zhang et al., 2009) extracts (Chinese) geographical information from language, where spatial relations are used for mapping to general categories of directional, topological, and distal relations using a rule-based system. Finally, in psycholinguistic studies, the important role of topological relations for expressing static spatial descriptions and even dynamic spatial configurations (Klippel and Li, 2009) and the frequent use of prepositions for topological relations (Kelleher et al., 2009) are highlighted.

\section{From Natural Language to Formal Spatial Relations}

Our approach for mapping spatial language to qualitative spatial formalizations consists of two levels: 


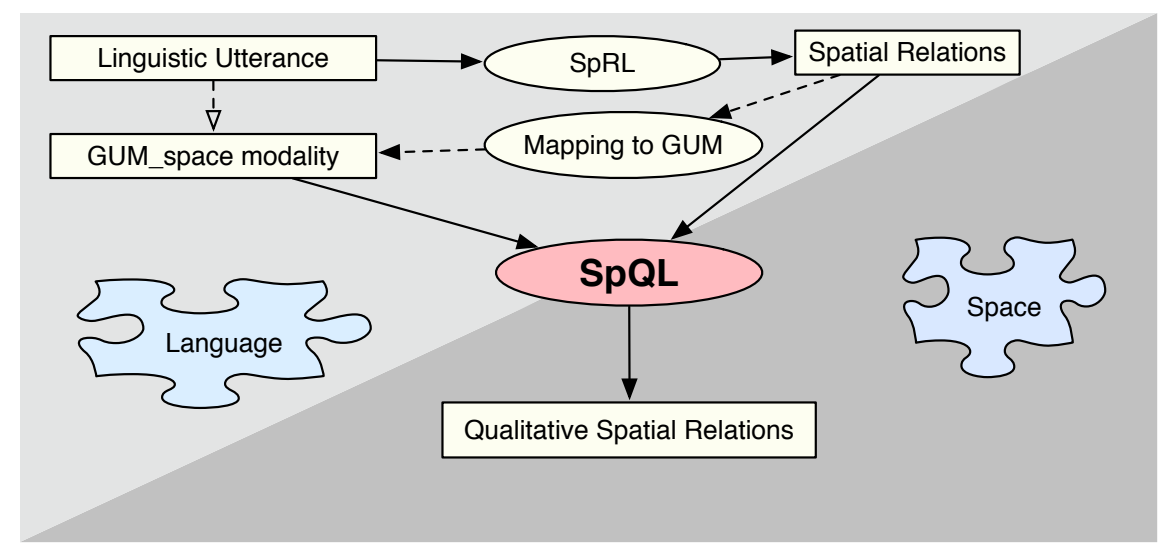

Figure 1: Mappings from language to spatial calculi.

(I) relevant linguistic features are selected and detected in natural language expressions, named spatial role labeling (SpRL), and

(II) these linguistic features are mapped to their 'interpretation' in a spatial calculus, named spatial qualitative labeling (SpQL).

Hence, we regard the feature analysis of spatial language as a separate level from a formal spatial representation (Bateman, 2010b), and for both levels we use machine learning techniques.

Figure 1 illustrates the two-level approach and it shows SpRL and SpQL anchored within the overall structure. The starting point is an utterance in natural language. In general, we aim at 'unrestricted' language, i.e., utterances contain arbitrary spatial and non-spatial information. One 
intermediate level consists of spatial relations extracted from the utterance. Such relations are automatically extracted from language (see Section 3.1.2). In addition, these extracted linguistic features can be classified according to modalities defined by the linguistically motivated ontology GUM-Space (Bateman et al., 2010). The ontological structure provides abstract linguistic constructs, of which the spatial modalities reflect specific types of spatial relations (see Section 3.1.3). On the basis of these linguistic analyses, a formalization process maps the spatial language to one or more qualitative spatial relations specified by qualitative spatial representations (QSR). These representations formalize types of spatial relations in a particular formal spatial system together with composition tables (see Section 3.2).

The distinction between the two levels in our approach "allows for the specification of a semantic representation that captures what the linguistic content of spatial expressions is committing to" (Bateman, 2010a); it separates the linguistic content from the formal spatial representation; and it provides a flexible way to map language to other spatial formalisms (Hois and Kutz, 2008b). Moreover, further components can be integrated, e.g., to support a situated interpretations or to support different types of uncertainties (Hois and Kutz, 2008a). An example of the overall process from the linguistic to the formal component is illustrated in Figure 2(a). 


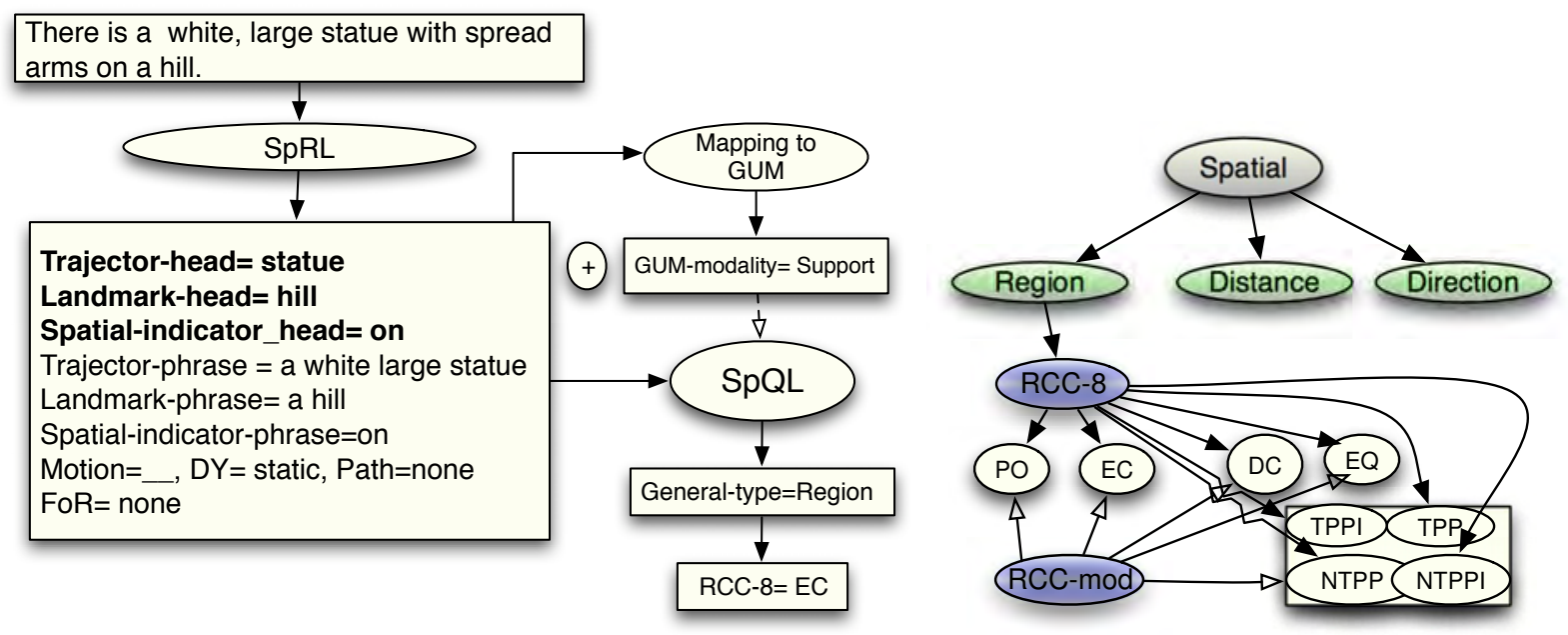

Figure 2: (a) An example sentence linguistically analyzed and mapped to QSR, (b) General and specific types of QSR. 
In the following, we discuss our computational approaches for both levels. We start with the spatial role labeling, i.e., the extraction of abstract spatial relations from language. This has been presented thoroughly in (Kordjamshidi et al., 2011b), and here, we (i) describe the task within our overall approach and (ii) show experimental results that demonstrate the feasibility of learning this mapping. Hereafter, we discuss the spatial qualitative labeling, i.e., the mapping from abstract spatial relations and GUM-Space modalities to qualitative spatial relations. This second level, which is the main contribution of this chapter, is demonstrated experimentally by using one specific qualitative spatial calculus, the RCC.

\section{1 (I) Linguistic Level: Extracting Spatial Relations from Language}

Spatial language has a broad but structured range of ways for relating spatial linguistic entities with each other, both semantically and syntactically (Bateman et al., 2010). This structure provides a classification of linguistic features, and it relates certain spatial roles to entities of a linguistic expression. These spatial roles (or features) can be used to relate linguistic expressions to abstract spatial formalizations, e.g., qualitative spatial calculi. To identify the spatial roles, we build on results from empirical and linguistic research about the way spatial language describes spatial information (Talmy, 2006; Zlatev, 2007), and we automatically detect and classify this 
spatial information in a sentence by using machine learning. Note, that our goal is to extract abstract spatial relations from language. For example, an abstract relation between book and table expressed by the preposition on can be detected by extracting the spatial roles of the individual words first in order to then classifying the relation on(book,table). The situational meaning of this abstract relation in terms of spatial semantics (e.g., in a topological sense) is detected by the second level in our approach, described in Section 3.2.

\subsubsection{Spatial Role Labeling}

We define spatial role labeling (SpRL) as the automatic labeling of words or phrases in sentences with a set of spatial roles. The roles take part in one or more spatial relations that are expressed by the sentence. The sentence-based spatial analysis of text deals with characterizing spatial descriptions, e.g., determining spatial properties of objects or their location. The spatial indicator identifies a spatial relation, and other constituents indicate participants in this relation. This is illustrated in the following example:

Give me $[\text { the gray book }]_{\text {trajector }}[\text { on }]_{\text {spatial_indicator }}[\text { the big table }]_{\text {landmark }}$.

The spatial role set of this sentence contains the spatial roles trajector for "the gray book", landmark for "the big table”, and spatial indicator for “on” (see (Zlatev, 2007; Kordjamshidi 
et al., 2010) for a discussion of these spatial roles). These and more spatial roles are distinguished and defined in the following way:

Trajector. The entity whose location or position is described. It can be static or dynamic; persons, objects, or events. Alternative common terms include local/figure object, locatum, referent, or target.

Landmark. The reference entity in relation to which the location or the motion of the trajector is specified. Alternate terms are reference object, ground, or relatum.

Spatial indicator. The element that defines constraints on spatial properties like the location of the trajector with respect to the landmark. A spatial indicator expresses the type of spatial relation and is often expressed by a preposition, but can also be a verb, noun, adjective, or adverb.

Motion Indicator. The element that provides specific spatial motion information. It is often expressed by verbs and defines the movement of a spatial entity, and renders a spatial expression dynamic instead of static.

Path. Start, intermediate, and end points can be expressed to provide path information for a movement, a re-orientation, or a location of an entity. 
Frame of Reference. In general, a frame of reference defines one or more reference points, and possibly a coordinate system based on axes and angles. Three reference types can typically be grammaticalized or lexicalized in English: intrinsic, relative, and absolute (Levinson, 2003). Recently, more detailed distinctions were presented in (Tenbrink and Kuhn, 2011), where spatial reference frames are represented and systematically specified by the spatial roles locatum, relatum, and (optional) vantage together with a directional system.

These six roles are the most influencing concepts for spatial semantics (Zlatev, 2007). SpRL can be considered as a special type of semantic role labeling, which is the task of automatically finding the semantic roles (e.g., agent, patient) for each predicate in a sentence. More specifically, this task determines the constituents in a sentence that are semantic arguments for a given predicate in order to determine appropriate roles for the arguments. Current approaches for semantic role labeling are based on supervised machine learning and require sufficient training and testing material (Màrquez et al., 2008). Similar to semantic role labeling, SpRL identifies and disambiguates the spatial indicator and its semantic role arguments, i.e., the trajector and landmark.

SpRL becomes more challenging the more complex a linguistic expression is, e.g., if an expression consists of several spatial relations. Even in the short example "The vase is on the 
ground on your left.", grammatical dependencies can cause errors with respect to spatial dependencies. The dependency parser relates the first appearance of "on" to the words "vase" and "ground", which produces a valid spatial relation. However, the parser relates the second appearance of "on" to the words "ground" and "your left", i.e., it produces an irrelevant spatial relation, namely "ground on your left". For more complex relations and nested noun phrases, the detection of 'spatially valid' relations is not straightforward and depends on the lexical meaning of words. Thus, recognizing the correct prepositional phrase (PP) attachment during syntactic parsing improves the identification of spatial features.

\subsubsection{Machine Learning for SpRL}

In SpRL we consider the spatial semantics that can be assigned to some words in the sentence as 'role-players'. We assume that the sentence is a priori partitioned into a number of segments (e.g.,

words, phrases). More formally, let $S$ be a sentence with $n$ segments $s=\left\langle w_{1}, w_{2}, \ldots, w_{n}\right\rangle$ and let the set of roles be ROLES $=\{$ TRAJECTOR, LANDMARK, SPATIAL_INDICATOR, MOTION_INDICATOR, PATH, FRAME_OF_REFERENCE, NONE $\}$, then each $w_{i} \in s$ needs to be labeled with one or more of these roles.

In (Kordjamshidi et al., 2011b), this task is formulated as a sequence tagging task and the 
triples TRAJECTOR, LANDMARK, and SPATIAL INDICATOR are extracted from the labeled sequences. A conditional random field model (CRF) (Lafferty et al., 2001) is used to tag single words in a sentence with the roles TRAJECTOR, LANDMARK, SPATIAL_INDICATOR, NONE. CRFs are instances of a large class of state-of-the-art machine learning models called probabilistic graphical models (Koller and Friedman, 2009), among which Bayesian networks are most well-known. A CRF represents input features and outputs (e.g., the role labels) as random variables, and represents a probability distribution over the joint occurrences of these variables using factors representing which features interact (i.e., correlate) and how. In several linguistic tasks, CRFs are used to model sequential relations, i.e., relations in which subsequent variables (e.g., words) correlate. In principle, however, any correlation between any set of features can be modeled and learned with CRFs.

Although the whole phrase in a sentence has a spatial role, we label only the head word of a phrase in SpRL with its spatial role. A head word is the word that determines the syntactic type of a phrase of which it is a member. The other elements of the phrase modify the head. For example, in "the huge blue book", the word "book" is the head word, and "huge" and "blue" are modifiers. Our annotated data assigns roles to both phrase and head word to maintain the flexibility for future experiments. For learning, we employ sequences containing exactly one 
(labeled) spatial indicator together with all related trajectors and landmarks. As a sentence can express multiple spatial relations, the sequence tagging extracts all of these relations:

Let $k$ be the number of prepositions in the sentence $s$, then $s$ gives rise to $k$ examples $e_{1} \ldots e_{k}$ where each $e_{i}(i=1 \ldots k)$ is a sequence $\left\langle\left(w_{1}, l_{1}\right), \ldots,\left(w_{n}, l_{n}\right)\right\rangle$ in which each word $w_{i}(i=1 \ldots n)$ is tagged as follows: i) at most one $w_{j}$ gets a label $l_{j}=$ SPATIAL_INDICATOR, ii) some words get a label TRAJECTOR or LANDMARK if they are trajector or landmark of the spatial indicator $w_{j}$, iii) the yet unlabeled words get the label NONE. In non-spatial sentences all words are tagged with NONE.

Here, we concisely list the linguistically motivated input features that are used for SpRL. These features are inspired by SRL, however, with the spatial indicator is the central feature. As mentioned above, spatial features are defined for head words only. For more details on extracting and labeling spatial features, see (Kordjamshidi et al., 2011b).

- Features of a word $w$ are defined by $f_{1}(w)$, which contains:

- the word (form) of $w$

- the part-of-speech tag

- the dependency to the syntactic head in the dependency tree 
- the semantic role

- the subcategorization of the word

- Features of the spatial indicator $s$ are defined by $f_{2}(s)$, which contains:

- the spatial indicator word (form)

- the subcategorization of $s$ (sister-nodes of its parent node in the tree)

- Relational features of $w$ wrt $s$ are defined by $f_{3}(w, s)$, which contains:

- the path in the parse tree from $w$ to $s$.

- the binary linear position of $w$ with respect to $s$ (e.g., before or not).

- The number of nodes on the path between $s$ and $w$ normalized by dividing over the number of all the nodes in the parse tree:

$$
\text { distance }=\frac{\text { \#Nodes on the path between } s \text { and } w}{\text { \#Nodes in the parse tree }}
$$

Table 1 illustrates two label sets for the spatial indicators "around" and "in".

If an appropriate number of sentences is labeled with information about the spatial indicator, trajector, landmark, motion indicator, path, and frame of reference, an automated spatial relation extraction method can be learned that can be employed successfully on new data. (Kordjamshidi 
Table 1: Example of two label sets for the sentence "A girl and a boy are walking around the desk in the classroom".

\begin{tabular}{|l|l|l|}
\hline Sentence & Label Set I & Label Set II \\
\hline girl & none & none \\
and & trajector & none \\
a & none & none \\
boy & trajector & none \\
are & none & none \\
walking & none & none \\
around & none \\
the & none & none \\
desk & landmark & none \\
in & nonecticator \\
\hline
\end{tabular}


Table 2: SpRL results on the extraction of triple relations (trajector, landmark, spatial indicator)

\begin{tabular}{|l|c|c|c|}
\hline Corpus Data & Precision & Recall & F-measure \\
\hline HCRC Map Task & 0.888 & 0.904 & 0.896 \\
ImageCLEF & 0.704 & 0.737 & 0.720 \\
\hline
\end{tabular}

et al., 2011b) showed that machine learning techniques can be used to provide automatic SpRL. Although the reader is referred to that work for the overall results and more details, Table 3.1.2 shows part of the results that we have obtained using CRF's. The evaluation is based on 10-fold cross-validation (see section 4.6.1 for more explanation). These results indicate adequacy of the learning based on two different corpora, namely selections from the HCRC Map Task corpus (Anderson et al., 1991) for spatial routes, and selections from the ImageCLEF IAPR corpus (Grubinger et al., 2006). The learning shows not only high performance for single spatial roles, such as trajector and landmark, but also good performance for the whole relation learning process. The whole relation means having the triples containing correct trajector, landmark and spatial indicator simultaneously.

Hence, the linguistic spatial roles can be learned with high enough reliability (precision and recall), and they can thus be used for the mapping to qualitative spatial relations. Also, 
information from lexical entries has a notable effect on the learning (cf. discussion on lexical impact in section 4), and automatic (semantic) generalizations and abstractions over the entities involved in the spatial relationships and their contextual implications may improve the learning and is a direction for further research in this area. Additional analyses demonstrate the adequacy of the employed features: an error analysis demonstrates that i) half of the errors are caused by misclassification of linguistic terms as none, ii) a quarter of the errors are caused by the complexity of nested spatial information, and iii) one-fifth of the errors are caused by irregularities in the grammar and focus shift in spatial descriptions. In the second step of SpQL, ground truth spatial role labels have been used as input for the mapping to spatial calculi.

\subsubsection{Linguistic Ontology}

In addition to the spatial features that are directly available from the linguistic surface, a formal semantic analysis can provide further support for the mapping from language to qualitative spatial representations (Pustejovsky et al., 2011; Zwarts, 2005; Eschenbach, 1999). This way, the lexical dependency can be reduced, and semantic categorizations can be used as an additional spatial feature in the mapping process. Here, we apply certain aspects taken from the spatial language categorization of the GUM-Space ontology, the Generalized Upper Model spatial 
extension (Bateman et al., 2010).

The GUM-Space ontology is a linguistically-motivated ontology that draws on findings from empirical cognitive and psycholinguistic research as well as on results from theoretical language science. It has been developed specifically for spatial language, in particular, for English and German. GUM-Space categorizes parts of natural language sentences that contain spatial information into groups that share the same semantics. For instance, every starting point of a motion (e.g., 'someone moving away from the door', 'leaving the house', or 'exiting the roundabout') is logically specified in the same way. In particular, GUM-Space is formalized as a description logic, which specifies spatial units that provide particular kinds of spatial information.

GUM-Space's spatial categorization is not based on lexical data, but on the way language characterizes spatial relationships either grammatically or inherently. It specifies information about trajector (LOCATUM or ACTOR), landmark (RELATUM), spatial indicator (MODALITY), motion indicator (MOTION), path (ROUTE), and the frame of reference (PERSPECTIVE), as defined for the SpRL above. Although routes and paths are further distinguished into subcategories in GUM-Space, we focus on the subcategorization of the spatial indicator (SPATIAL MODALITY), as we have only few dynamic examples in our experiments below.

Figure 3 shows the 73 spatial modalities (i.e., spatial indicators) that are distinguished in the 


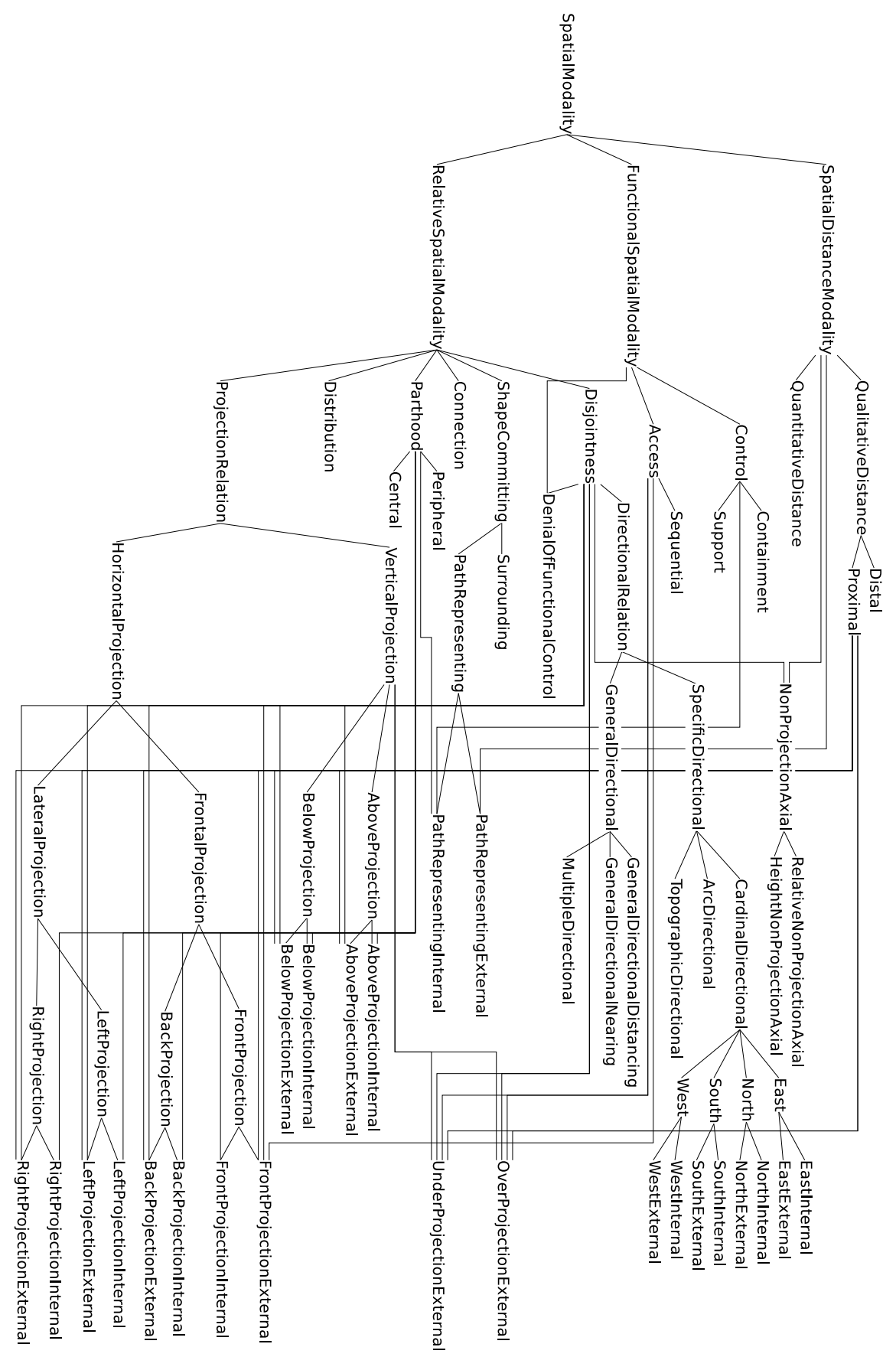

Figure 3: GUM-Space's spatial modalities. 
GUM-Space ontology based on their hierarchical dependencies (Bateman et al., 2010). 60 of these modalities can be expressed in natural language directly, the other modalities are generalized supertypes that provide more structure to the categorization. The modalities are not constrained to specific lexical entries, and they provide a distinction of spatial modes of locatings according to their lexico-grammatical expression as well as their semantic implications. Thus, these modalities add further semantic information about the spatial indicator, i.e., the spatial relationship between the trajector and landmark. For example, the specific spatial modality expressed by "on" in the sentence "The vase is on the ground" is SUPPORT, which defines a physical supportive locating of another entity, i.e., if the landmark moves the trajector moves as well (Tyler and Evans, 2003).

Spatial modalities can be computationally specified and accessed by using categorical grammar techniques (Bateman et al., 2010). In the following, however, we focus on two aspects with regard to the GUM-Space modalities: first, we investigate if the fine-grained classification of the spatial modalities can be learned also by using machine learning techniques based on the linguistic features extracted from the SpRL; second, we reuse the resulting spatial modality to map linguistic expressions to qualitative spatial relations. As illustrated in Figures 1 and 2(a), GUM-Space is used as an intermediate semantic representation between the linguistic features and the qualitative spatial representations. This is motivated by the fine-grained distinction of 
spatial modalities that reflect the type of spatial relation between trajector and landmark, which is one of the the strongest indicators for the qualitative relation. For this purpose, the corpus data used to learn the mapping to spatial qualitative relations (see Section 4.2) contains annotations for spatial modalities. These are used together with the other linguistic features during the learning process for the mapping to qualitative representations. The results show an improvement in the mapping performance when using spatial modalities as a learning feature to map linguistic data to a selected spatial calculus (see Section 4.6).

\section{2 (II) Spatial Level: Mapping Linguistic Features to Qualitative Spatial}

\section{Relations}

We denote the mapping process from linguistic features to qualitative spatial representations as the spatial qualitative labeling (SpQL), and define it as the automatic mapping of the output of SpRL and GUM-Space to qualitative spatial representations (spatial calculi). Not only ambiguity and underspecification of spatial information in natural language but also over-specification of qualitative spatial calculi make a direct mapping between the two sides difficult (Bateman, 2010b). Most of the qualitative spatial calculi focus on a single aspect, e.g., topology, direction, distance, or shape (Renz and Nebel, 2007). Spatial language, however, often conveys multiple 
meanings within one expression (Carlson and Van Deman, 2004). Hence, the SpQL has to cover multiple aspects with a practically acceptable level of generality. Nevertheless, obtaining an annotated corpus is the main challenge to use machine learning for the SpQL, and we built such a corpus in this work. Essential for such corpora is not only that they contain a large sufficiently large number of spatial language texts for learning but also that they have additional (non-linguistic) spatial information such as images or maps, from which a qualitative spatial model can be built that can be related to the textual information. These constraints hold for the selected corpora that we use in our experiments.

To build a corpus that supports supervised machine learning models to cope with these challenges, (Kordjamshidi et al., 2010) propose an annotation framework inspired by SpatialML (Mani et al., 2008) and a related scheme presented in (Shen et al., 2009). Using the training data which is annotated based on that scheme, we map the linguistic features to a general spatial type. The general spatial type is primarily expressed by the spatial indicator on the linguistic surface. In our current version, the available general spatial types are REGION, DIRECTION, and DISTANCE as these are primarily distinguished by spatial information (Renz and Nebel, 2007). For future work further types will be relevant, such as SHAPE, GRADUATION, or SIZE. However, the three general spatial types cover the coarse-grained aspects of space, for 


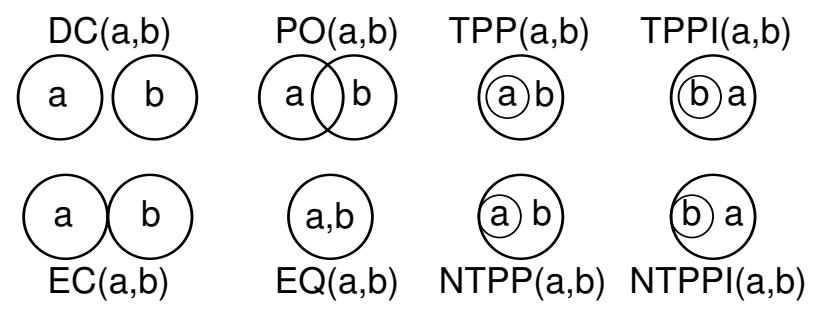

Figure 4: The RCC-8 relations.

which qualitative spatial calculi are available. In general, every spatial indicator can be associated with one ore more of these general types. After mapping the linguistic features to the general spatial type, we map the linguistic features to a specific spatial type, which is the qualitative relation available by the spatial calculus used in the mapping process. Figure 2(b) above illustrates the distinction between general and specific types. For example, if the general spatial type is detected as REGION, a specific spatial type of a region-based qualitative spatial calculi is detected next.

In the $\mathrm{SpQL}$ experiments presented in Section 4.6, we map linguistic corpus data to the Region Connection Calculus RCC-8 (Randell et al., 1992), which reflects region-based topological relations as introduced below. Using RCC-8 in our learning experiments is motivated by several reasons: the linguistic descriptions in the corpus data express primarily static spatial configurations of objects located with regard to a topological relation to another object based on 
2-D images; topology is "probably the single most important spatial concept fundamental to cognition in general" (Klippel, 2011); topological or region-based spatial information has been researched in depth in the area of qualitative spatial representation and reasoning; although RCC-8 only formalizes static qualitative relations between regions, its neighborhood graph provides a method to model movement patterns and spatial change over time (Freksa, 1991), i.e., to model dynamic spatial configurations in terms of RCC-8; we assume that the trajectors and landmarks can often be interpreted as spatial regions and, as a consequence, their relation can be annotated with a specific RCC-8 relation.

The last argument is also important, as it allows us to map the linguistic features directly to qualitative spatial relations. For RCC-8, we can thus define which linguistic terms are interpreted as qualitative entities, namely the trajector is interpreted as a region, the landmark is interpreted as a region, and the spatial indicator is interpreted as a qualitative relation. Although our approach is technically able to map linguistic data to other qualitative representations or more elaborate types of spatial formalizations, we expect that more input knowledge will be required to achieve an acceptable learning performance. This additional input knowledge may be based on external resources or ontological information, however, such experimental analyses and the construction of related annotated corpus data are left for future work. 
RCC- 8 provides 8 basic relations (see Figure 4): disconnected DC(a, b), externally connected $\mathrm{EC}(\mathrm{a}, \mathrm{b})$, partial overlap $\mathrm{PO}(\mathrm{a}, \mathrm{b})$, equal $\mathrm{EQ}(\mathrm{a}, \mathrm{b})$, tangential proper-part $\mathrm{TPP}(\mathrm{a}, \mathrm{b})$, non-tangential proper-part $\operatorname{NTPP}(a, b)$, tangential proper-part inverse $\operatorname{TPPI}(\mathrm{a}, \mathrm{b})$, and non-tangential proper-part inverse $\operatorname{NTPPI}(a, b)$, which describe mutually exclusive and exhaustive overlap and touching relationships between two well-behaved regions in space.

As our machine learning for the SpQL maps the entire set of spatial features from a sentence to a specific spatial model, we gain a more flexible and powerful mapping, in contrast to mapping only the single spatial indicator to an RCC-8 relation. Furthermore, the SpQL can map linguistic features to more than one calculus (e.g., to RCC-8 and an orientation calculus), which supports the required level of expressivity for a spatial linguistic expression. In the long run, such annotations will enable to use expressive (probabilistic) logical models that relate linguistic spatial features of a sentence to complex configurations of spatial relations expressed in several spatial calculi simultanously. Learning this multiple mapping can also be used to visualize the spatial semantic contents of the sentence, or - when viewed as probabilistic constraints of these contents - to reason about most likely inferences that can be drawn. For example, from a sentence "the mouse is next to the keyboard on the table", one might infer the distance between these objects to be around 20 centimeters based on external knowledge about table sizes and typical 
configurations of the objects and one might also infer the angulation and positions of the objects with regard to the table. Some of our current investigations concern expressive logical models in a learning setup along the lines of the approach we discuss here; this is beyond the scope of this chapter and discussed as future work direction in Section 5.

In summary, we investigate the following aspects in this work:

Given adequate corpus annotations, is it feasible to map linguistic spatial features to spatial calculi models, are the basic relations of the calculi clearly distinguishable by using the linguistic features, are similar sets of features mapped to similar basic relations, are some basic qualitative relations hardly available in the corpus, and which linguistic features do we need for an acceptable classification?

As mentioned above, RCC-8 is far less expressive than natural language, e.g., when comparing its 8 basic relations with the 73 spatial modalities semantically distinguished in GUM-Space. As a result, the SpQL leads to loss of information or to a simplification by the qualitative model. However, our experiments show that the mapping process is non-trivial and most of the basic relations can be distinguished clearly with high learning performance when spatial role labels are known. 


\section{Machine Learning: From Spatial Language to RCC}

In this section, we present the machine learning task for mapping linguistic spatial features extracted from natural language to spatial calculi (see again Figure 1). In particular, we map spatial language to the region-based calculus RCC-8. For this purpose, we use a general machine learning approach, in which the linguistic features from the linguistic level are described through feature vectors that form the input to the system. The output consists of a label derived from RCC-8 for every qualitative spatial relation. This means mapping to one of eight labels from RCC-8 $=\{$ EC,DC,TPP,TPPI,NTPP, NTPPI,PO,EQ,NONE $\}$ (see also Figure 4). We also describe additional experiments in which we map to 5 labels from a modified RCC-8 and in which we use the (60 linguistically expressible) spatial modalities from GUM-Space. Note, that the main purpose of our experiments is to show that the mapping from language to space can indeed be learned. For a general analysis, larger amounts of corpus data annotated with several spatial calculi would be necessary. As discussed in Section 3.2, we limit our experimental setup to map the linguistic data to RCC-8 relations for practical and theoretical reasons. However, the experimental results still provide enough information to examine the feasibility of learning qualitative spatial relations from a corpus. 


\subsection{Preliminaries}

The main hypothesis to test is whether a mapping can be learned from extracted spatial roles and relations (SpRL) to specific models of formal QSR (SpQL). This amounts to learning the real semantics of these abstract roles and relations from annotated data, i.e., interpreted as qualitative spatial types. The spatial roles with a high influence on this task are TRAJECTOR, LANDMARK, and SPATIAL_INDICATOR. Our central experimental investigation is whether detected trajectors, landmarks, and spatial indicators, are adequate for determining the specific type of qualitative spatial relations.

In (Kordjamshidi et al., 2011b) (and Section 3.1 on spatial role labeling), it was shown that these elements can be effectively extracted from natural language by using machine learning. Here, we investigate the learnability question for several fine- or coarse-grained versions of region-based QSR, namely RCC-8 and a corpus-inspired modification of it. We also investigate if the spatial roles MOTION, PATH, and FRAME OF REFERENCE have an effect on this learning process, although motion and path are more applicable for dynamic spatial relations and frame of reference is relevant in projective relations. As most of our data set contains static topological relations, we expect these roles to have almost no influence on the learning for the SpQL. Furthermore, we investigate the influence of GUM-Space spatial modalities in the mapping 
process. A general problem in many linguistic tasks (using machine learning) is the lack of lexical information in the training data, i.e., the sensitivity to specific words. Naturally, we encounter this problem in our approach, in both SpRL and SpQL. Hence, we conducted additional inter-corpora evaluations particularly for SpRL in (Kordjamshidi et al., 2011b) to investigate induced errors and to gain insights concerning possible abstractions required for future work.

\subsection{Data Sets}

As supervised machine learning requires annotated data, our approach relies on spatial linguistic and qualitative corpus data. As, to our knowledge, this data is not publicly available so far, we have built a corpus based on the annotation scheme in (Kordjamshidi et al., 2010) and spatial annotations of GUM-Space. Our corpus set consists of textual descriptions of 613 images taken from the IAPR TC-12 Image data set (Grubinger et al., 2006), referred to as CLEF. It induces 1213 English sentences and 1716 corresponding spatial relations. CLEF contains images taken by tourists with descriptions in several languages, describing depicted objects and their absolute and relative positions. This makes it a rich resource for spatial information. However, the descriptions contain non-spatial descriptions of images, which makes it a useful corpus for general, less domain-specific approaches. In addition to CLEF, we annotated and used 64 selected sentences 
from the Map Task corpus (which has been used to validate the expressivity of spatial relations in GUM-Space (Bateman et al., 2010)) and 124 sentences from the Room Description corpus (Shi and Tenbrink, 2009). The more detailed results are reported for CLEF, as it is a reasonably large data set.

The textual descriptions have been indexed and annotated with spatial roles of trajector, landmark, and their corresponding spatial indicator. Separate roles are assigned to phrases and the head words of the phrases. The verbs and verb phrases are indexed and annotated, particularly when they participate in forming the spatial configurations, and this is mostly the case for dynamic spatial relations. Moreover there are annotations for path in terms of beginning, middle, or end. Each sentence with a spatial relation is annotated as DYNAMIC or STATIC, and each spatial relation is annotated with a GUM-Space modality. Moreover, every sentence with a spatial relation is annotated with general and specific spatial types (see Section 3.2). We have measured the inter-annotator agreement for 325 sentences with respect to the spatial roles trajector, landmark, and spatial indicator. With two annotators, the Kappa value resulted in 0.896 with a 95\% confidence interval (0.882-0.910).

Our CLEF data set contains 1040 annotated topological, 662 directional, and 91 distance relations. In the current corpus only 50 examples are annotated with more than one general spatial 
type. For example, "next to" is annotated as a topological relation DC in terms of RCC-8 and as a distance relation CLOSE in terms of a relative distance calculus:

(7) Two people are sitting next to her.

trajector: people,

landmark: her,

spatial indicator: next to,

general type: region/distance,

specific type: RCC-8 / relative-distance,

spatial value: DC / close,

DY: static,

path: none,

frame of reference: none,

spatial modality: Proximal

\subsection{D vs. 3D Annotations}

Although the textual data used is accompanied by images (CLEF) or maps (Map Task and Room Description), the qualitative spatial annotations for CLEF was based on the text itself. This was done to focus on information that can actually be extracted from the language itself. Nevertheless, 
human imagination about a described scene can interfere with the textual description, which has resulted in some variations. As an example, take the following sentence and its annotation:

(8) Bushes and small trees (are) on the hill.

trajector: bushes,

landmark: the hill,

spatial indicator: on,

general type: region,

specific type: RCC-8,

spatial value: EC,

DY: static,

path: none,

frame of reference: none,

spatial modality: Support

This 3-D projection of the description of a 2-D image is annotated as externally connected. In the

2-D image, however, a partial overlap may also be adequate. In contrast, a 2-D map (with an

allocentric perspective) of the described scene would lead to a non-tangential proper part

annotation. This example illustrates what we have said before; that RCC-8 alone is - quite 
naturally - not enough to capture adequately all necessary spatial information, and that in a more general approach more (and combinations of) qualitative spatial calculi have to be used.

\subsection{Dynamic vs. Static Annotations}

In the CLEF data set 25 of the relations are annotated as DYNAMIC, the others as STATIC. If a dynamic situation is annotated with a (static) RCC-8 relations, the qualitative relation can be regarded as a snapshot of the situation. This is shown in the following example:

(9) People are crossing the street. 
trajector: people,

landmark: road,

spatial indicator: crossing,

general type: region / direction,

specific type: RCC-8 / undefined,

spatial value: EC / undefined,

DY: dynamic,

path: middle,

frame of reference: none,

spatial modality: PathRepresentingInternal

Hence, the annotations refer to time slices for the (linguistic) explanation of the (static) image. This allows a mapping from dynamic descriptions to (static) RCC-8 relations mainly by including the path feature and the relative situation of the trajector with respect to an imaginary path related to the landmark. Allowing RCC-8 annotations for dynamic descriptions is also supported by the conceptual neighborhood graphs (Freksa, 1991): Every topological change, i.e., movements of regions with respect to each other and their changing relations, can be split into a sequence of adjacent RCC-8 relations according to the neighborhood graph (Klippel and Li, 2009). The annotated RCC-8 relation thus reflects one relation out of this sequence, i.e., one moment in time 
of the topological change. However, we may not predict if the annotations refer to a time slice that reflects the start, intermediate, or end point of the path or the motion process. For instance, (Regier and Zheng, 2007) show that linguistic expressions seem to focus primarily on the end point of the motion.

\subsection{Features and Experimental Setup}

In the first set of experiments we apply machine learning techniques to learn an automatic mapping from SpRL to the following RCC relations.

RCC-8. RCC-8=\{EC, DC, TPP, TPPI, NTPP, NTPPI, PO, EQ, NONE $\}$

RCC-mod. RCC-MOD $=\{\mathrm{EC}, \mathrm{DC}, \mathrm{PP}, \mathrm{PO}, \mathrm{EQ}, \mathrm{NONE}\}$, i.e., subsuming $\{\mathrm{TPP}, \mathrm{NTPP}, \mathrm{TPPI}$, NTPPI $\}$ under $\{P P\}$

The features that are used as the input of the SpQL are the head words that are annotated as TRAJECTOR (tr), LANDMARK (lm), SPATIAL INDICATOR (sp), and MOTION INDICATOR (mo); features that include not only head words but all words in the phrase are TRAJECTOR PHRASE (trPh), LANDMARK PHRASE (lmPh), and SPATIAL INDICATOR PHRASE (spPh); the PATH FEATURE (pa) has one of the values $\{$ BEGINNING, MIDDLE, END $\}$; the frame of reference (for) 
Table 3: Feature count for CLEF.

\begin{tabular}{|l|l|l|l|l|l|l|l|l|l|l|l|}
\hline tr & $1 \mathrm{~lm}$ & $\mathrm{sp}$ & $\operatorname{trPh}$ & $\operatorname{lmPh}$ & $\mathrm{spPh}$ & $\mathrm{mo}$ & $\mathrm{pa}$ & for & dy & gum & Total \\
\hline 354 & 183 & 43 & 258 & 142 & 56 & 12 & 4 & 2 & 2 & 39 & 1095 \\
\hline
\end{tabular}

has one of the values \{INTRINSIC, RELATIVE, ABSOLUTE\}; the dynamicity feature (DY) has one of the values \{STATIC, DYNAMIC ; and the GUM-Space modality (gum) has one of its 60 possible values (see Figure 3). An example sentence with its linguistic features is shown in Figure 2(a). The occurrences of these features in CLEF are shown in Table 3. These features are gradually used to model the learning process, and the performance of the learning is measured by gradually adding linguistic features to evaluate their effect on the learning process. The prior distribution of the RCC-8 relations in CLEF (the main data set) and the other two corpora is shown in Table 4. We applied various machine learning approaches, however, best results were obtained with support vector machines (SVM), as described in the following. An SVM is a state-of-the-art machine learning technique typically used for classification and regression problems. It represents examples as points in space and learns to find a boundary in this space that optimally separates positive from negative examples of a concept. New examples are then mapped to the same space and are labeled as the side of the boundary to which they belong (cf. (Alpaydin, 2004)). The 
Table 4: RCC-8 annotations of CLEF, Map Task, and the Room Description corpus.

\begin{tabular}{|l|l|l|l|}
\hline RCC-8 Relations & \#Instances(CLEF) & \#Instances(Map Task) & \#Instances(Room Des.) \\
\hline DC & 147 & 32 & 82 \\
\hline TPP & 369 & 3 & 2 \\
\hline NTPP & 12 & 3 & 0 \\
\hline EC & 458 & 2 & 1 \\
\hline EQ & 5 & 0 & 5 \\
\hline NTPPI & 1 & 0 & 0 \\
\hline TPPI & 25 & 0 & 0 \\
\hline PO & 15 & 69 & 34 \\
\hline NONE & 684 & 29 & 124 \\
\hline Total & 1716 & 69 & 0 \\
\hline
\end{tabular}


second set of experiments examines the mapping from linguistic features to GUM-Space spatial modalities and to general qualitative spatial types. This is technically implemented in the same way, and the learning model is evaluated by gradually adding input features.

\subsection{Results and Discussion}

In this section, the results of the spatial qualitative labeling (SpQL) to RCC-8 and RCC-mod are discussed, and the influence of the linguistic features for the SpQL task is analyzed. Also the feature impact on the mapping to general qualitative spatial types and the effect of using GUM-Space modalities is presented.

\subsubsection{RCC-8}

First, we investigate the feasibility of automatically mapping the linguistic features to RCC-8 relations. For this purpose, several classifiers (e.g., decision trees, naive Bayes, and SVM) were used. The results in Table 5 present the accuracy in terms of precision, recall, and F-measure over individual classes and the weighted average overall for the best performing classifier (SVM) using 10-fold-cross-validation. This means that the data set is divided into 10 parts. Each training time leaves out one of the 10 parts as the evaluation set and the model is trained on the remaining 9 
parts. The overall performance measures are the averages over all 10 parts used for evaluation. Precision, recall, and F-measure are computed using:

$$
\begin{gathered}
\text { recall }=\frac{T P}{T P+F N} \quad ; \quad \text { precision }=\frac{T P}{T P+F P} \quad ; \quad \text { F-measure }=\frac{2 * \text { precision } * \text { recall }}{\text { precision }+ \text { recall }} \\
\text { with } \quad \mathrm{TP}=\# \text { correctly classified samples wrt the target } \mathrm{RCC} \text { relation } \\
\mathrm{FN}=\# \text { samples incorrectly not assigned to the target } \mathrm{RCC} \text { relation } \\
\mathrm{FP}=\# \text { samples incorrectly assigned to the target } \mathrm{RCC} \text { relation }
\end{gathered}
$$

F-measure is the harmonic average of precision and recall (van Rijsbergen, 1979). Table 5 shows that the weighted average in which the performance of each class multiplied by the number of its instances (i.e micro averaging) is reasonably high. For classes with more examples in the training data the performance is higher. The performance over none-RCC-8 relations is rather strong. The performance for DC, EC, TPP, and PO is reasonably high, however for the other relations it is less acceptable.

\subsubsection{RCC-mod}

When humans use language to describe objects, mostly the salient objects are chosen as landmarks in the descriptions. The inverse proper part relations thus occur less frequently 
Table 5: RCC-8 classification results for CLEF.

\begin{tabular}{|l|l|l|l|l|}
\hline RCC-8 Relation & \#Instances & Precision & Recall & F-measure \\
\hline DC & 147 & 0.848 & 0.871 & 0.859 \\
\hline TPP & 369 & 0.801 & 0.897 & 0.847 \\
\hline NTPP & 12 & 0.667 & 0.50 & 0.571 \\
\hline EC & 458 & 0.859 & 0.841 & 0.850 \\
\hline EQ & 5 & 0.8 & 0.8 & 0.8 \\
\hline NTPPI & 1 & 0 & 0 & 0 \\
\hline TPPI & 25 & 0.778 & 0.28 & 0.412 \\
\hline PO & 15 & 0.778 & 0.467 & 0.583 \\
\hline NONE & 684 & 0.970 & 0.953 & 0.962 \\
\hline Total(Weighted Avg.) & 1716 & 0.886 & 0.886 & 0.883 \\
\hline
\end{tabular}


(cf. (Klippel and Li, 2009)). This motivates the next experiment in which we combine all variations of proper part including $\{\mathrm{TPP}, \mathrm{NTPP}, \mathrm{TPPI}, \mathrm{NTPPI}\}$ into one class $\{\mathrm{PP}\}$, resulting in RCC-mod (for RCC-mod, however, no spatial reasoning is directly available). Table 6 shows the results over the five classes of RCC-mod in addition to the 'none' class. Although, the overall performance for both RCC-8 and RCC-mod classification is high, there are still two critical relations, namely EQ and PO, with only few examples in the data set. The confusion matrix indicates that most errors are caused by EC in both experiments, in particular, by 2-D object-related distinctions, e.g.:

(10) “A ship on the see...", which is annotated as PO

(11) “A bag on the table...”, which is annotated as EC

The classifier could not distinguish between these two examples and classifies both of them as EC. This happens because in the corpus most of these cases are annotated with EC and there are not enough examples to distinguish PO.

The relation EQ too is a challenging relation and cannot easily be inferred and described by analyzing respective 2-D images. The equality would mean that two areas have a full overlap, i.e., one completely covers the other and vice versa. This will often not be recognizable from 2-D 
Table 6: RCC-mod classification results for CLEF.

\begin{tabular}{|l|l|l|l|l|}
\hline RCC-mod Relation & \#Instances & Precision & Recall & F-measure \\
\hline DC & 147 & 0.863 & 0.857 & 0.86 \\
\hline EC & 458 & 0.858 & 0.856 & 0.857 \\
\hline EQ & 5 & 0.75 & 0.6 & 0.667 \\
\hline PP & 407 & 0.838 & 0.887 & 0.862 \\
\hline PO & 15 & 0.778 & 0.467 & 0.583 \\
\hline NONE & 684 & 0.975 & 0.953 & 0.964 \\
\hline Total(Weighted Avg.) & 1716 & 0.899 & 0.898 & 0.898 \\
\hline
\end{tabular}


images and consequently will not be explicitly expressed in the linguistic utterance. However, EQ occurs for five relations in our corpus, and by looking at these examples it can be observed that there is a high potential of disagreement across the annotations. An example is the sentence “... with his hands in his pockets", which has been annotated as EQ although it could as well be annotated as PP in RCC-mod.

\subsubsection{Feature Analysis}

The idea of exploiting the above mentioned features is inspired by cognitive linguistics and generally derived from the main elements of spatial semantics in spatial language. Here, we present an empirical study to investigate the influence of each of these features on the performance of the machine learning models for CLEF. We performed two sets of experiments using the RCC-8 classification setting and we looked at the weighted average of all classes with the same SVM classifier.

In the first experiment (Figure 6), the spatial indicator is used as the only input feature for classification of the RCC-8 relations. Spatial indicators are mostly prepositions in CLEF, and using only them for the mapping yields an F-measure of 0.474. Adding the head word of the

landmark phrase in the sentence increases the F-measure by 0.27 . Gradually adding the head 


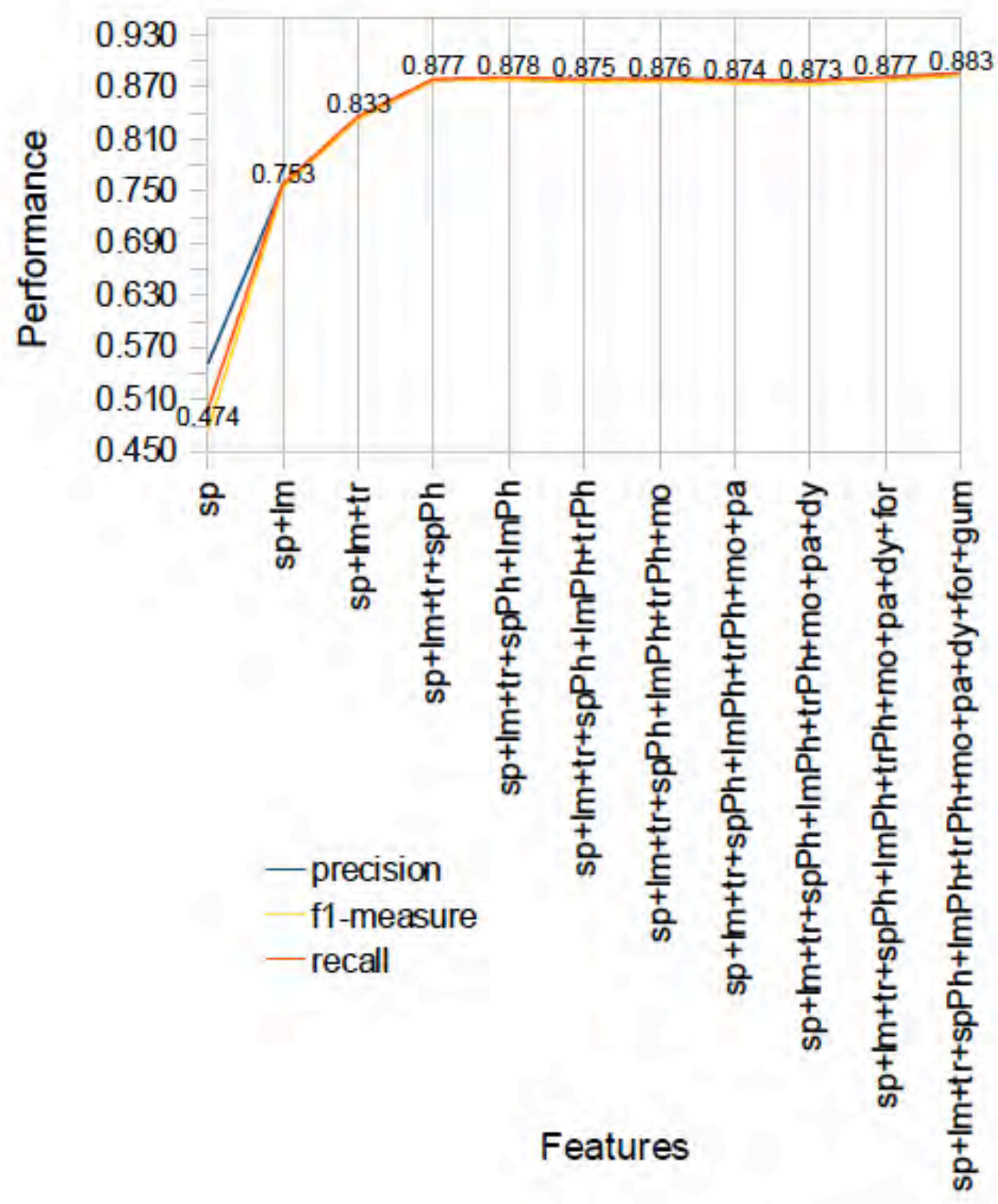

Figure 5: Performance of the RCC-8 classifier with spatial roles as input. 


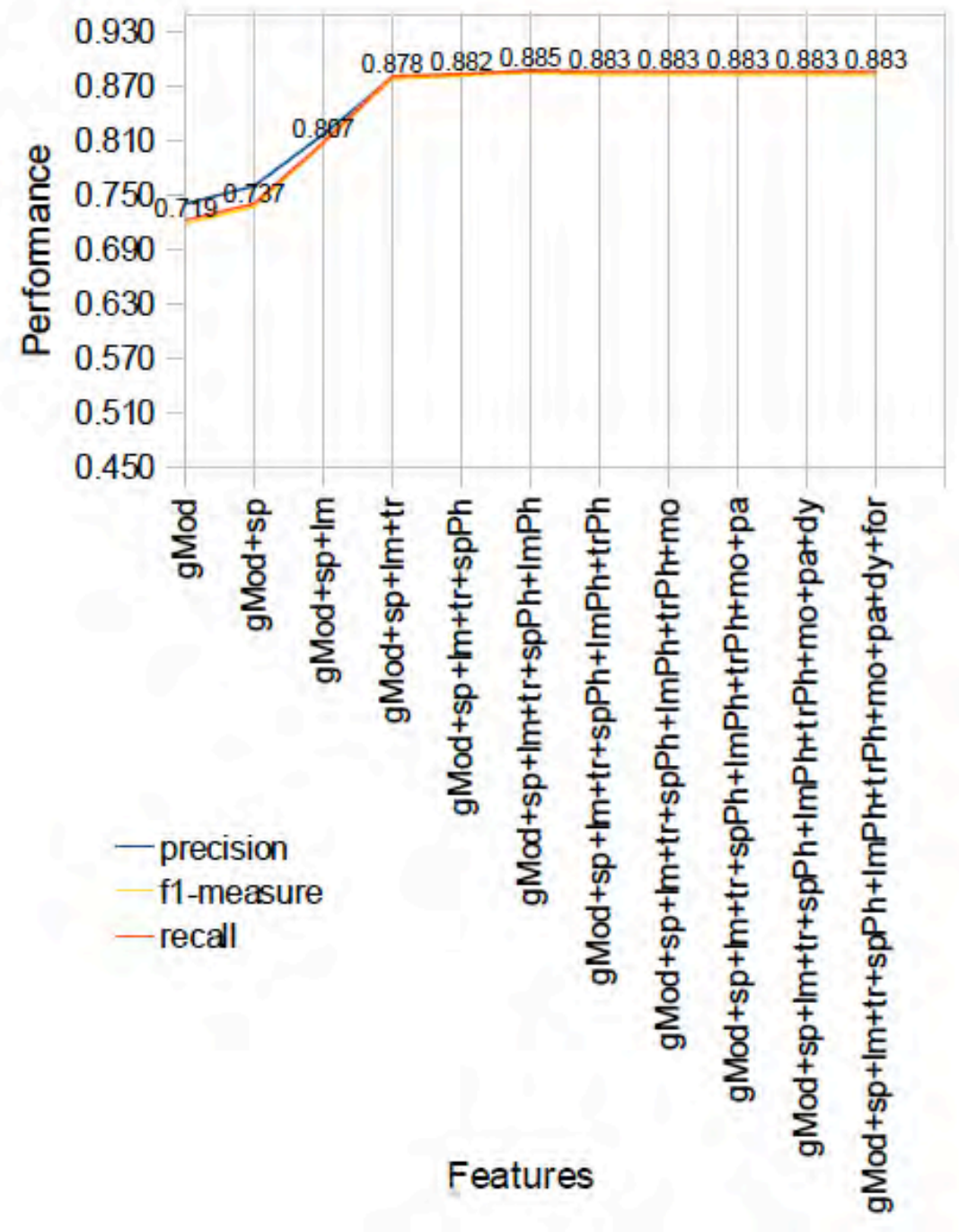

Figure 6: Performance of the RCC-8 classifier with GUM-Space modalities and spatial roles as input. 
word of the trajector adds another 0.08. Adding additional features increases again the performance by 0.044 , and accuracy becomes stable. Further feature additions may sometimes even reduce performance due to the combination of noise and lack of data.

In the second experiment (Figure 5), we use GUM-Space modality information as the first step, motivated by the high expressivity of GUM-Space and its in-depth exploration with regard to the spatial relations expressed in spatial language. Using only GUM-Space's spatial modalities yields an F-measure of 0.719. Given the spatial modality, the spatial indicator (mostly the preposition) does not add more significant information and the performance is increased by 0.02 . However, the context has an impact on the performance: adding the landmark information increases the performance by 0.07 ; Adding the trajector information gives a 0.07 increase. The remarkable outcome of this experiment is that given the modality feature even trivial fluctuations in the accuracy of the classifiers are not observed anymore, and the model seems to behave more robustly against extra and noisy features.

Although trajector and landmark information may not carry explicit spatial indications in terms of QSR relations, using these features increases the overall performance in both experimental setups. This may particularly be caused by the statistical significance for choosing certain reference objects over others, which is shown in Barclay and Galton (this volume). For 
instance, absolute sizes of trajector and landmark are important for selecting the reference objects in the initial CLEF annotations of the photographs.

As we use GUM-Space modalities only with manual annotations so far, the third experiment (Figure 7) investigates whether the modalities can also be learned by using the automatically identified spatial roles. Using only the spatial indicator, the performance is 0.55 and thus slightly above chance. However, using also landmark information increases performance to 0.82 , which goes up to 0.96 when using the whole phrase of the spatial indicator. This feature influence seems reasonable as the modalities reflect spatial relations with regard to their landmarks. The experiment shows that indeed spatial modalities can be learned for this data set, however, only 21 from the 60 modality types were used for the annotations.

Adding the phrasal information of the trajector has no effect or even some minor negative effects on the overall performance. In Table 6, the 5 EQ instances are classified with 0.8 accuracy when using all features; when reducing these features F-measure decreases by 0.4 . Reducing the features, however, has a reverse effect on the PO relation and an increase of 0.11 is obtained, caused by the lack of sufficient data for EQ and PO.

Besides the phrasal information the other sets of features have no significant impact on the performance. According to the reported statistics over the applied data set, this result is plausible 


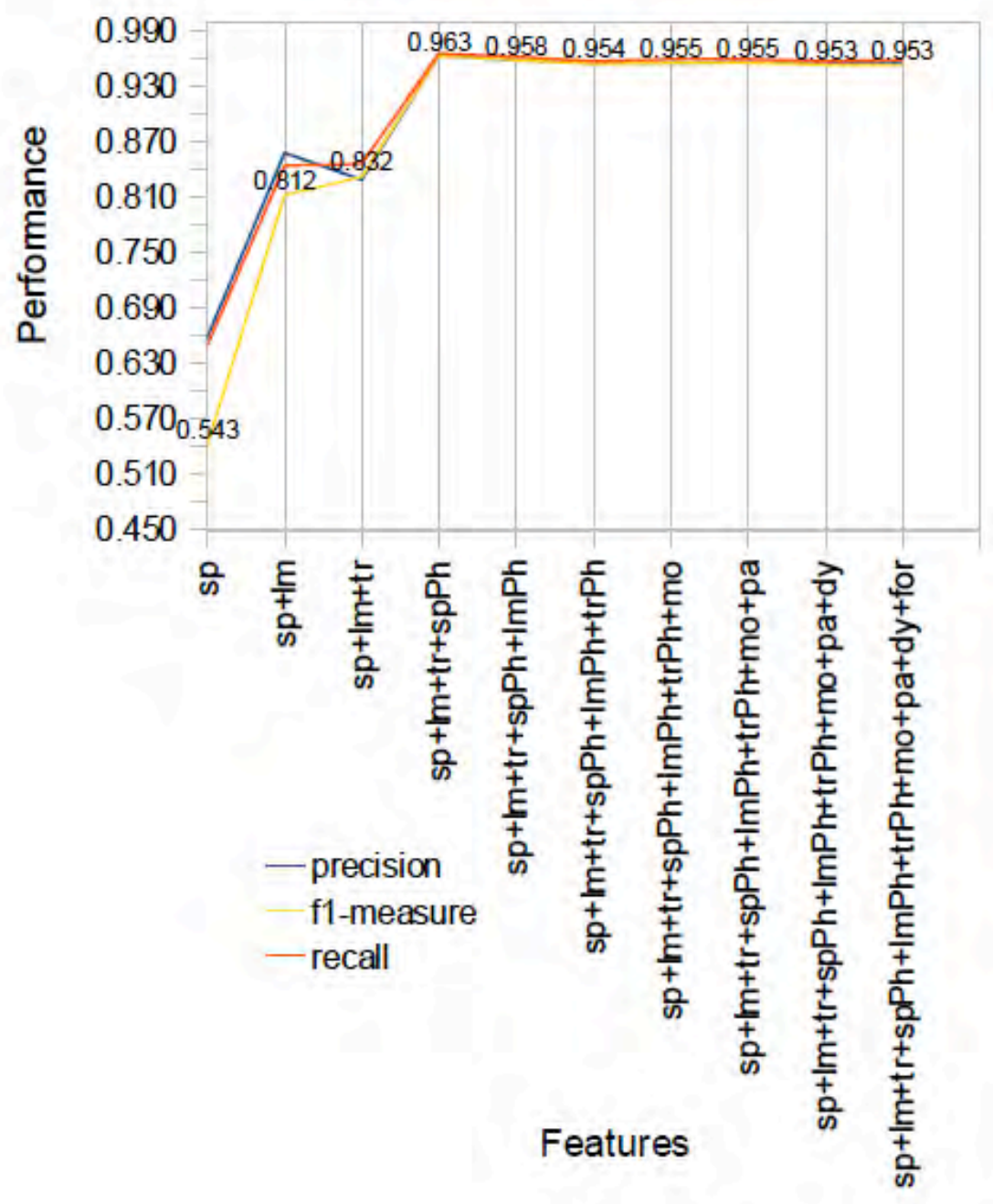

Figure 7: GUM-Space modality classification. 
because CLEF contains mostly static relations, and only few motions are annotated in the corpus. Hence, dynamicity and path features do not occur often in the data and are not discriminative features for the type of the RCC-8 relations. The frame of reference is often annotated as NONE, as it is not available for topological relations. Consequently, this feature did not have an effect on the performance. However, even for directional relations that are based on a frame of reference, this feature might often be ambiguous when given implicitly (see Avraamides et al., this volume).

\subsubsection{Mapping to General Categories of Spatial Relations}

In addition to region information, direction and distance relations are relevant for mapping spatial language to QSR (cf. Figure 2). This can be considered as a first step towards the SpQL to various types of calculi. As we pointed out before, a spatial relation at the linguistic level can be mapped to various types of calculi since it can be assigned to more than one of these general categories. As discussed above, the expression "next to" implies distance and topology information.

Figures 8 and 9 present this accuracy in terms of input features, using either the spatial modality or the spatial indicator as starting point. As the number of distance relations in the data set is only 32, a lower performance than for topological relations is expected. Similar to the previous experiments, the most influential features are trajector, landmark, spatial indicator, and 


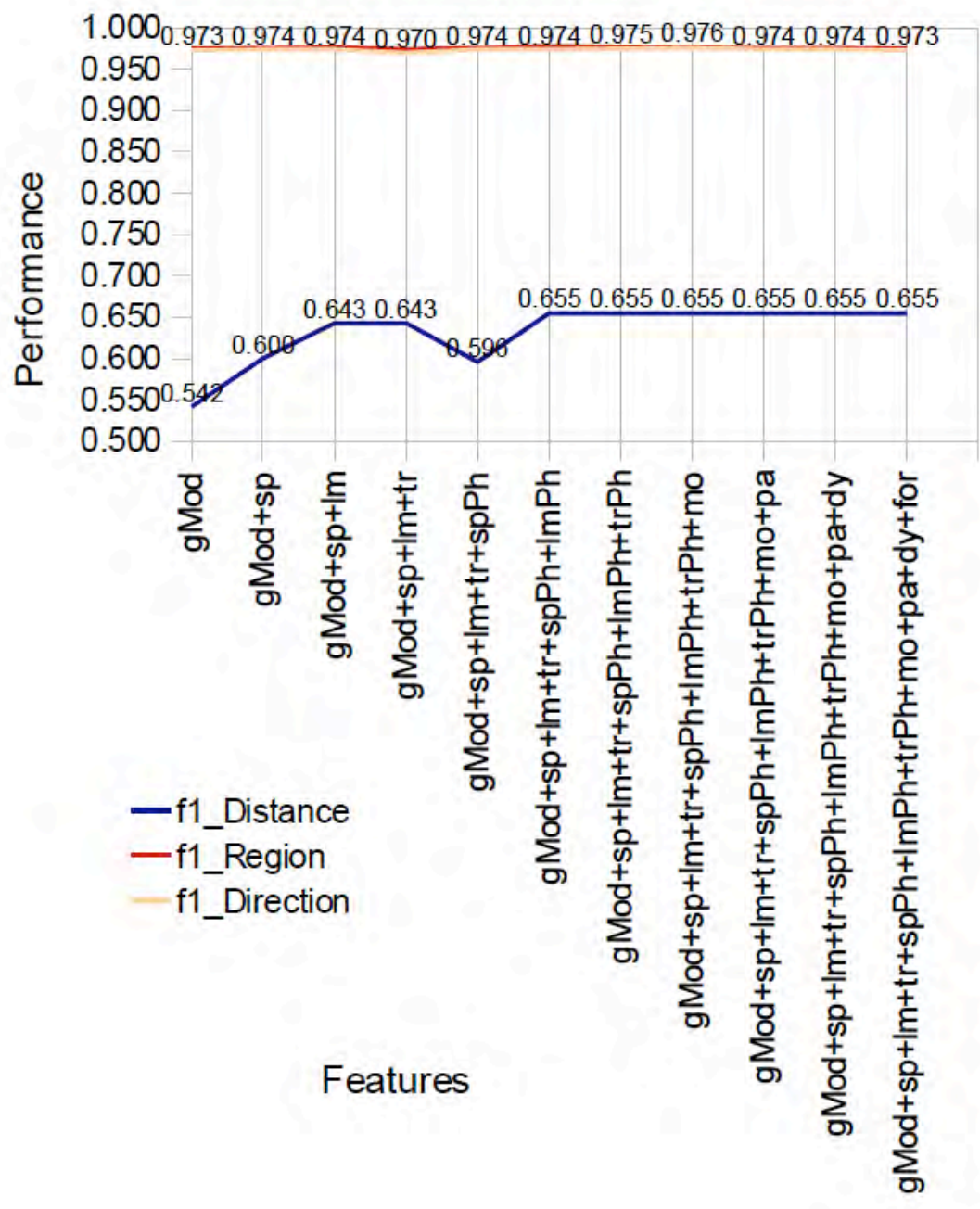

Figure 8: Performance of the general spatial type classifier starting with spatial modality as input feature. 


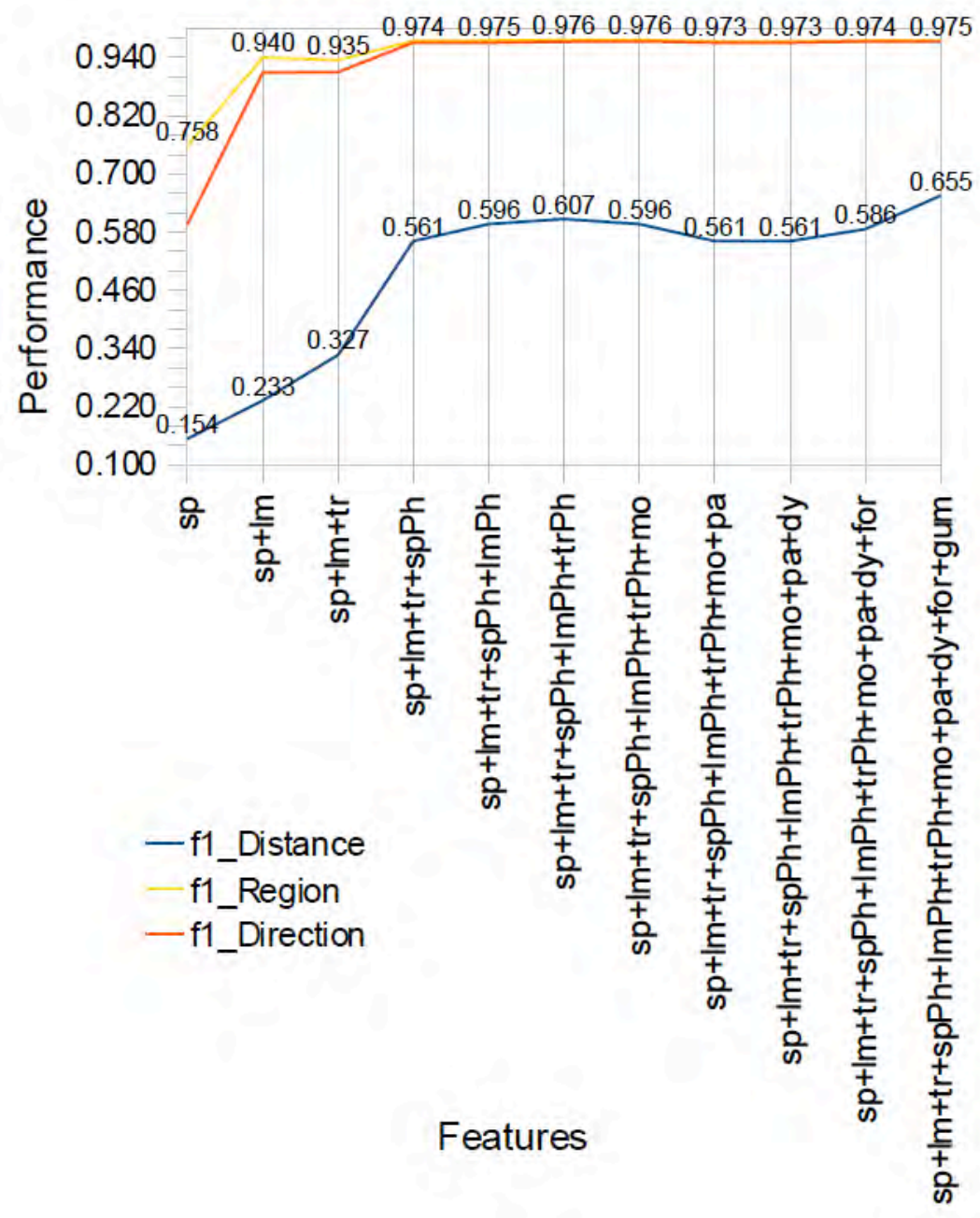

Figure 9: Performance of the general spatial type classifier starting with spatial indicator as input feature. 
the phrase of the spatial indicator. Due to the low number of examples, the positive effect of the features is more distinct. Using information of the spatial modality again improves the final performance: knowing the spatial modality for the relation, the general qualitative spatial type is almost certain and the remaining features do not affect this result (cf. Figure 8). This result is most likely caused by the specific category types of GUM-Space, which directly imply the general type. However, the confusion matrix shows that distance relations are less well recognized, again due to lack of examples in the data set.

\subsubsection{Inter-Corpora Evaluation}

Even though we use SpRL as an abstract linguistic representation, lexical information has a notable effect on the SpQL. Lexical information specifically from trajectors and landmarks is used for learning the spatial roles in the previous experiments, which did not use abstraction mechanisms, such as word classes. In this section, we examine the influence of lexical information for the two other corpora Map Task and Room Description.

As shown in Table 4, the two corpora lack several types of topological relationships, because they contain mostly directional relations for route instructions on a map or in a room and their relations are annotated mostly as DC in terms of RCC-8 or as NONE. In several tests, we did the 
same 10-fold cross validation internally on this data sets. Here, the F-measure on both was higher than $70 \%$, which is acceptable with respect to the small data sets.

In another experiment we used the trained machine learning model for CLEF and used it on those two corpora, which resulted in low performance. The main reason of this outcome is that the lexical entries for the objects described in these two corpora are entirely different from CLEF's lexical entries.

\section{Conclusions and Future Work}

The aim of this work was to investigate whether a mapping from complex spatial utterances to formal spatial relations can be learned. We have introduced a two-level machine learning architecture that consists of SpRL (spatial role labeling) to extract and identify spatial linguistic features based on a holistic approach and SpQL (spatial qualitative labeling) to map these features to qualitative spatial relations such as RCC.

Our experimental results show, most importantly, that indeed both the extraction of spatial features and the mapping to qualitative relations can be learned, and that the approach is computationally feasible. Feature analysis indicates that the most influential features are trajector, 
landmark, and spatial indicator. Although phrase information seems to have no effect on the learning, it might become more informative when the phrase contains additional functional information. As we mapped primarily static and region-based spatial descriptions in our experiments, the spatial features path, motion, and frame of reference had a negligible effect on the results. An outcome with regard to RCC-8 mappings is that some qualitative relations did hardly occur in the (random) corpus data set (e.g., inverse and equality relations).

The use of formal semantic categories from GUM-Space was useful to improve the performance of SpQL. As GUM-Space aims at classifying the linguistic types of spatial semantics in a structured way, it appears to be adequately expressive for SpQL, in particular, when mapping to general spatial relation types. Using the GUM-Space modalities, learning becomes more robust when lexical information is fluctuating across the data set. As a consequence, we expect the modalities to have a positive effect when mapping to qualitative relations other than RCC-8. Our experiments showed that it is promising to use the linguistic features for learning the GUM-Space modalities, as these are highly compatible with the linguistic spatial patterns in SpRL. However mapping to the whole GUM's ontological information and learning the ontological categories remains future work.

Another future direction is the mapping to more than one qualitative spatial representation, 
including calculi for regions, orientations, distances, shapes, sizes, motion, etc. As spatial language is able to express all of these types and combinations thereof, the SpQL task should be able to interpret a linguistic expression more comprehensively when having multiple spatial calculi at hand. Given SpQL, the mapping to multiple calculi is expected to be easy to accomplish with relational machine learning. So far, we have employed so-called propositional representations in our learning approaches like SVM and CRF. More expressive relational (also called first-order logical) representations too can be used in the learning process, which are more natural for representing spatial information as it is linguistically expressed in a relational way. Statistical relational learning approaches (De Raedt et al., 2008) combine such representations with state-of-the-art algorithms (e.g., based on the same principles as probabilistic graphical models or SVMs) to exploit logical representations while dealing with uncertainty and ambiguity in natural language. In current and future work we investigate these models for spatial language understanding.

Our inter-corpora evaluations identified challenges with respect to lack of lexical information when the learned model was used in other domains. This rather unsurprising aspect could be compensated in several ways. The data set can be easily extended by integrating diverse sources, which is, however, a time-consuming and costly approach. The use of abstractions of 
lexical information seems to be more applicable, e.g., abstract features that indicate functional characteristics of trajectors and landmarks may improve the mapping to qualitative spatial relations. This will add another layer of abstract labels for the linguistic data. The annotation types of these abstract labels could be taken from WordNet or word taxonomies, or latent word models could be used. The latent word language model proposes words that can replace the trajector and landmark words so that the set of training examples can be automatically expanded by these replacements (Deschacht and Moens, 2009).

In conclusion, this chapter has shown a machine learning approach and an empirical study for interpretation of spatial natural language in terms of QSR. As long as adequate corpus data exists, we expect that the approach is able to learn respective qualitative relations with an acceptable performance. The kind of spatial language interpretation should be particularly applicable in several domains, whenever a mapping from language to abstract spatial representations are required. Examples are dialogue systems (Kruijff et al., 2007), spatial assistance system for design or media production (Bhatt et al., this volume), text-to-scene conversion systems (Rouhizadeh et al., 2011), virtual human cooperations (Nguyen and Wachsmuth, this volume), and robotic navigation instructions (Kollar et al., 2010). 


\section{References}

Alpaydin, E. (2004), Introduction to Machine Learning. The MIT Press, Cambridge, Massachusetts.

Anderson, A.H.; Bader, M.; Bard, E.; Boyle, E.; Doherty, G.; Garrod, S.; Isard, S.; Kowtko, J.; McAllister, J.; Miller, J.; Sotillo, C.; Thompson, H.; and Weinert, R. (1991), 'The HCRC Map Task Corpus.' Language and Speech, 34(4), pp. 351-366.

Bateman, J. (2010a), 'Situating spatial language and the role of ontology: issues and outlook.' Linguistics and Language Compass, 4(8), pp. 639-664.

Bateman, J.A. (2010b), 'Language and Space: a two-level semantic approach based on principles of ontological engineering.' Int. J. of Speech Technology, 13(1), pp. 29-48.

Bateman, J.A.; Hois, J.; Ross, R.; and Tenbrink, T. (2010), 'A linguistic ontology of space for natural language processing.' Artificial Intelligence, 174(14), pp. 1027-1071.

Carlson, L.A. and Van Deman, S.R. (2004), 'The space in spatial language.' J. of Memory and Language, 51, pp. 418-436. 
De Raedt, L.; Frasconi, P.; Kersting, K.; and Muggleton, S. (eds.) (2008), Probabilistic Inductive Logic Programming. LNCS, Springer.

Deschacht, K. and Moens, M.F. (2009), 'Semi-supervised semantic role labeling using the latent words language model.' In Empirical Methods in Natural Language Processing, EMNLP'09, pp. 21-29.

Eschenbach, C. (1999), 'Geometric structures of frames of reference and natural language semantics.' Spatial Cognition and Computation, 1(4), pp. 329-348.

Fonseca, F. and Rodriguez, M.A. (2007), 'From Geo-Pragmatics to Derivation Ontologies: New Directions for the GeoSpatial Semantic Web.' Transactions in GIS, 11(3), pp. 313-316.

Freksa, C. (1991), 'Qualitative Spatial Reasoning.' In D. Mark and A. Frank (eds.), Cognitive and Linguistic Aspects of Geographic Space, pp. 361-372, Kluwer Academic Publishers, Dordrecht.

Grubinger, M.; Clough, P.; Müller, H.; and Deselaers, T. (2006), 'The IAPR Benchmark: A New Evaluation Resource for Visual Information Systems.' In Int. Conf. on Language Resources and Evaluation, LREC'06. 
Hois, J. and Kutz, O. (2008a), 'Counterparts in Language and Space - Similarity and $\mathcal{S}$-connection.' In Formal Ontology in Information Systems, FOIS'08, pp. 266-279.

Hois, J. and Kutz, O. (2008b), 'Natural Language meets Spatial Calculi.' In Spatial Cognition VI: Learning, Reasoning, and Talking about Space, pp. 266-282.

Kelleher, J.; Sloan, C.; and Namee, B.M. (2009), 'An investigation into the semantics of English topological prepositions.' Cognitive Processing, 10(2), pp. 233-236.

Kelleher, J.D. and Costello, F.J. (2009), 'Applying Computational Models of Spatial Prepositions to Visually Situated Dialog.' Computational Linguistics, 35(2), pp. 271-306.

Klippel, A. (2011), 'Spatial Information Theory Meets Spatial Thinking - Is Topology the Rosetta Stone of Spatial Cognition.' Annals of the Association of American Geographers, to appear.

Klippel, A. and Li, R. (2009), 'The Endpoint Hypothesis: A Topological-Cognitive Assessment of Geographic Scale Movement Patterns.' In Spatial Information Theory, COSIT’09, pp. 177-194.

Kollar, T.; Tellex, S.; Roy, D.; and Roy, N. (2010), 'Toward understanding natural language directions.' In 5th ACM/IEEE Int. Conf. on Human-Robot Interaction, HRI'10, pp. 259-266.

Koller, D. and Friedman, N. (2009), Probabilistic Graphical Models: Principles and Techniques. MIT Press. 
Kordjamshidi, P.; Hois, J.; van Otterlo, M.; and Moens, M.F. (2011a), 'Machine Learning for Interpretation of Spatial Natural Language in terms of QSR.' Poster Presentation at the 10th International Conference on Spatial Information Theory (COSIT'11).

Kordjamshidi, P.; van Otterlo, M.; and Moens, M.F. (2010), ‘Spatial Role Labeling: Task Definition and Annotation Scheme.' In 7th Conf. on Int. Language Resources and Evaluation, LREC'10.

Kordjamshidi, P.; van Otterlo, M.; and Moens, M.F. (2011b), 'Spatial Role Labeling: Towards Extraction of Spatial Relations from Natural Language.' ACM Transactions on Speech and Language Processing, Accepted under minor revision.

Kruijff, G.J.M.; Zender, H.; Jensfelt, P.; and Christensen, H.I. (2007), 'Situated dialogue and spatial organization: What, where. . and why?' Int. J. of Advanced Robotic Systems, 4(1), pp. $125-138$.

Kurata, Y. and Shi, H. (2008), 'Interpreting Motion Expressions in Route Instructions Using Two Projection-Based Spatial Models.' In KI 2008: Advances in Artificial Intelligence, pp. 258-266.

Lafferty, J.; McCallum, A.; and Pereira, F. (2001), 'Conditional Random Fields: Probabilistic Models for Segmenting and Labeling Sequence Data.' In 18th Int. Conf. on Machine Learning, 
ICML'01, pp. 282-289.

Levinson, S.C. (2003), Space in Language and Cognition: Explorations in Cognitive Diversity. Cambridge University Press.

Li, H.; Zhao, T.; Li, S.; and Zhao, J. (2007), 'The Extraction of Trajectories from Real Texts Based on Linear Classification.' In NODALIDA 2007 Conf., pp. 121-127.

Mani, I.; Hitzeman, J.; Richer, J.; Harris, D.; Quimby, R.; and Wellner, B. (2008), ‘SpatialML: Annotation Scheme, Corpora, and Tools.' In 6th Int. Language Resources and Evaluation, LREC'08.

Màrquez, L.; Carreras, X.; Litkowski, K.C.; and Stevenson, S. (2008), 'Semantic Role Labeling: An Introduction to the Special Issue.' Computational Linguistics, 34(2), pp. 145-159.

Pustejovsky, J.; Moszkowicz, J.; and Verhagen, M. (2011), 'ISO-Space: The Annotation of Spatial Information in Language.' In ACL-ISO International Workshop on Semantic Annotation (ISA'6).

Randell, D.A.; Cui, Z.; and Cohn, A.G. (1992), 'A Spatial Logic Based on Regions and Connection.' In 3rd Int. Conf. on the Principles of Knowledge Representation and Reasoning, KR'92, pp. 165-176. 
Regier, T. and Zheng, M. (2007), 'Attention to Endpoints: A Cross-Linguistic Constraint on Spatial Meaning.' Cognitive Science, 31(4), pp. 705-719.

Renz, J. and Nebel, B. (2007), 'Qualitative Spatial Reasoning Using Constraint Calculi.' In M. Aiello; I. Pratt-Hartmann; and J. van Benthem (eds.), Handbook of Spatial Logics, pp. 161-215, Springer.

Rouhizadeh, M.; Bauer, D.; Coyne, B.; Rambow, O.; and Sproat, R. (2011), 'Collecting Spatial Information for Locations in a Text-to-Scene Conversion System.' In J. Hois; R. Ross; J. Kelleher; and J. Bateman (eds.), Computational Models of Spatial Language Interpretation and Generation (CoSLI'11), pp. 16-23, CEUR-WS Proceedings.

Roy, D. and Reiter, E. (2005), 'Connecting Language to the World.' Artificial Intelligence, 167(1-2), pp. 1-12.

Shen, Q.; Zhang, X.; and Jiang, W. (2009), 'Annotation of Spatial Relations in Natural Language.' In Int. Conf. on Environmental Science and Information Application Technology.

Shi, H. and Tenbrink, T. (2009), 'Telling Rolland where to go: HRI dialogues on route navigation.' In K. Coventry; T. Tenbrink; and J. Bateman (eds.), Spatial Language and Dialogue, pp. 177-189, Oxford Univ. Press. 
Talmy, L. (2006), 'The fundamental system of spatial schemas in language.' In B. Hampe (ed.), From Perception to Meaning: Image Schemas in Cognitive Linguistics, pp. 37-47, Mouton de Gruyter.

Tenbrink, T. and Kuhn, W. (2011), 'A model of spatial reference frames in language.' In M. Egenhofer; N. Giudice; R. Moratz; and M. Worboys (eds.), Conference on Spatial Information Theory (COSIT'11), pp. 371-390, Springer.

Tyler, A. and Evans, V. (2003), The Semantics of English Prepositions: Spatial Scenes. Embodied Meaning and Cognition, Cambridge University Press, Cambridge.

van Rijsbergen, C.J. (1979), Information Retrieval. Butterworths.

Zhang, C.; Zhang, X.; Jiang, W.; Shen, Q.; and Zhang, S. (2009), 'Rule-Based Extraction of Spatial Relations in Natural Language Text.' In Int. Conf. on Computational Intelligence and Software Engineering.

Zlatev, J. (2007), 'Spatial Semantics.' In D. Geeraerts and H. Cuyckens (eds.), The Oxford Handbook of Cognitive Linguistics, pp. 318-350, Oxford Univ. Press.

Zwarts, J. (2005), 'Prepositional aspect and the algebra of paths.' Linguistics and Philosophy, 28(6), pp. 739-779. 